\title{
Picosecond electrical switching of single-gate metal nanotip arrays
}

\author{
Soichiro Tsujino ${ }^{\text {a) }}$ \\ Laboratory for Micro- and Nanotechnology, Department of Synchrotron Radiation and Nanotechnology, \\ Paul Scherrer Institut, CH-5232 Villigen-PSI, Switzerland
}

\author{
Martin Paraliev \\ Advanced Accelerator Division, Department of Large Research Facilities, Paul Scherrer Institut, \\ CH-5232 Villigen-PSI, Switzerland
}

(Received 26 August 2013; accepted 11 November 2013; published 10 December 2013)

\begin{abstract}
Electrical switching of single-gate all metal field emitter arrays is studied to generate subnanosecond electron pulses. By applying a bipolar current pulse method to the metal nanotip array, electron pulses with the duration down to 210 ps were generated. To explore the short-pulse limit of the proposed switching method, the propagation of the gate potential across the array was analyzed by numerical simulation. The result shows that single-gate field emitter arrays with the array diameter of $1 \mathrm{~mm}$ can be electrically switched with the duration down to $\sim 5$ ps. (c) 2014 American Vacuum Society. [http://dx.doi.org/10.1116/1.4838295]
\end{abstract}

\section{INTRODUCTION}

Pulsed field emission current can be generated from single-gate field emitter arrays (FEAs) by applying a switching potential in the order of $100 \mathrm{~V}$. The duration of the field emission pulses is normally limited by the gate capacitance between the electron extraction gate electrode and the emitter as well as the resistance of the substrate. In order to inject electron pulses with subnanosecond bunching structure into high gain traveling-wave tube (TWT) radio-frequency (RF) amplifiers, Whaley et al. developed a Spindt FEA with low substrate resistance and gate capacitance reduced down to $20 \mathrm{pF}{ }^{1}$ Using such a FEA, they demonstrated experimentally an emission-gated operation of a TWT up to $7 \mathrm{GHz}{ }^{1}$ Recently, a different approach was used to generate subnanosecond electrically gated electron pulses. ${ }^{2,3}$ In these studies, FEAs with relatively high gate capacitance $(200-400 \mathrm{pF})$ but with small substrate resistance below $\sim 10^{-3} \Omega$ (Fig. 1) were used. The FEAs were gated by applying a pair of short current pulses with opposite polarity as shown in Fig. 2. In this way, electron emission was turned on and off with the duration approximately given by the separation $T_{\mathrm{s}}$ between the two current pulses. The rise time and the fall time of the gate potential are determined by the amplitude and the duration of the first and the second injection current pulses, respectively. The field emission pulse duration $T_{\mathrm{c}}$ takes a value somewhat smaller than $T_{\mathrm{s}}$ because of the nonlinearity of the field emission current-voltage characteristic. This bipolar current pulse switching method was used at the combined pulsed diode-RF accelerator of the SwissFEL gun teststand facility at the Paul Scherrer Institute ${ }^{2,3}$ in order to test the compatibility of FEAs with high acceleration electric field and measure the transverse beam emittance. Also, by combining the initial pulsed acceleration with the following acceleration in RF cavities, the FEA electron beam was accelerated up to $5 \mathrm{MeV}{ }^{2}$ However, large energy spread was observed, because the FEA emission pulse was longer than

a)Electronic mail: soichiro.tsujino@psi.ch one half of the RF acceleration cycle, equal to $333 \mathrm{ps}$; the pulse duration of the FEA pulse was evaluated to be equal to 390 ps from the RF phase dependent overlap of the field emission pulse. ${ }^{4,5}$ Therefore, an order of magnitude shorter FEA pulses are required to utilize these FEAs as electron sources for accelerator applications such as free-electron lasers. Although the intrinsic emittance of single-gate FEAs, equal to $\sim 1 \mathrm{~mm} \operatorname{mrad}$ for a $1 \mathrm{~mm}$ diameter FEA, $, 4,6$ is an order of magnitude larger than double-gate FEAs, ${ }^{7,8}$ single-gate FEAs with picosecond switching time are advantageous compared to thermionic emitters gated by grid electrode. ${ }^{9,10}$

In this work, we explore the limitation of the bipolar current pulse method for generating short field emission pulses from all metal FEAs. Using the proposed method in a DC gun teststand, ${ }^{6}$ field emission pulses with the duration well below $1 \mathrm{~ns}$ were produced. A numerical simulation was used to analyze the limit of this switching method on the field emission pulse duration by the gate potential propagation delay of the gate potential pulse across the FEA.

\section{FEA FABRICATION AND EXPERIMENT}

We fabricated a single-gate FEA by a molding method and a self-align gate fabrication method. ${ }^{11}$ The FEA consisted of $10^{5}$ molybdenum nanotip emitters supported on a $300-\mu$ m-thick electroplated nickel substrate. The emitters were pyramidal shape with $1.5 \mu \mathrm{m}$ square base, distributed over an area with the diameter $D_{\text {FEA }}$ equal to $2.2 \mathrm{~mm}$ with $5 \mu \mathrm{m}$ pitch as shown in Fig. 1. The radii of the curvature of the emitter tip apexes were $5-10 \mathrm{~nm}$. The gate electrode was fabricated from a $0.5-\mu$ m-thick molybdenum film supported by a $1.2-\mu$ m-thick $\mathrm{SiO}_{2}$ gate insulator layer.

The FEA chip (square with $7 \mathrm{~mm}$ side) was mounted in a low-inductance FEA holder, designed to enable the picosecond electrical switching shown in Figs. 3 and 4. The holder is also compatible with acceleration electric field up to $\sim 100 \mathrm{MV} / \mathrm{m}^{2,3,12,13}$ The switching pulse is applied to the FEA substrate $(E m)$ via a coaxial transmission line and an N-type coaxial connector. The center conductor of the 

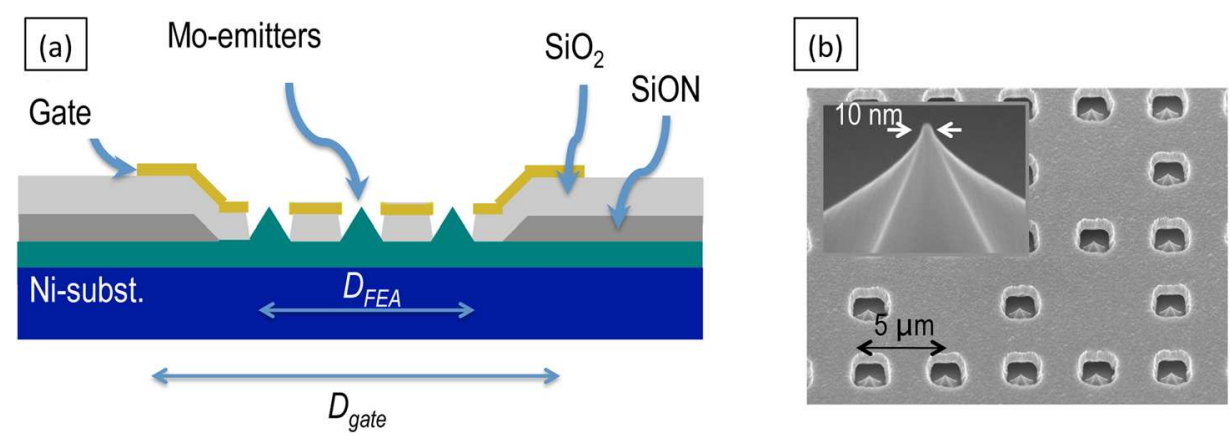

FIG. 1. (Color online) (a) Schematic cross-section of all metal FEA. (b) SEM image of a 5- $\mu$ m-pitch FEA used in the experiment.

coaxial transmission line is extended by a spring-loaded contact that presses the back of Em. The return electrical contact to the gate electrode $\left(G_{\mathrm{ex}}\right)$ is created by placing the FEA chip on the metal lip at the inner edge of the FEA holder ${ }^{13}$ (Fig. 3). The opening above the FEA is $4 \mathrm{~mm}$ in diameter. The FEA is exposed to the acceleration field of the gun through this opening. At the FEA surface, the electric field is reduced by $\sim 20 \%$ from the value at the FEA holder surface at $z=1 \mathrm{~mm}$ because of a recess of the FEA from the cathode face by $1 \mathrm{~mm}$, see Fig. 4 . Due to the finite dimension of the opening, the acceleration electric field varies across the FEA surface as well. With the 4-mm-diameter opening, the field decreases toward the periphery by $5 \%$ and $1 \%$, respectively, for 2.2- and 1-mm-diameter arrays. The total gate diameter ( $D_{\text {gate }}$ in Fig. 1) is $4.5 \mathrm{~mm}$ in order to avoid exposing the gate insulator surface and the triple-point to the acceleration electric field. To partially compensate the gate capacitance increase by taking this extra gate area, we increased the insulator thickness to $2.4 \mu \mathrm{m}$ over the region outside of the array, by inserting a 1.2- $\mu$ m-thick SiON layer [see Fig. 1(a)]. Due to this FEA design modification, the total gate capacitance of the FEA was reduced from $\sim 0.4 \mathrm{nF}$ to $0.2-0.3 \mathrm{nF}$. The bipolar current pulse was generated by differentiating a voltage pulse with $\sim 400$ ps FWHM (full width at the half maximum) duration with the amplitude of $\sim 1 \mathrm{kV}$ delivered by a $50 \Omega$ matched short pulse generator (FPM 5-1PM, FID GmbH, Germany ${ }^{14}$ ).

The pulse experiment was conducted in the DC gun teststand, Fig. 5. The original design and construction of the gun was reported previously. ${ }^{6}$ An acceleration potential $V_{\text {acc }}$ of $-(20-100) \mathrm{kV}$ is applied to the cathode. The instruments for the FEA switching are located in an insulated high voltage cabinet; a regulated high voltage power supply charges the cathode and the insulated cabinet to $V_{\text {acc }}$, while the anode is connected to ground. Alternatively, it is possible to operate the gun by applying a DC potential $(\sim 1 \mathrm{kV})$ to the anode block while keeping the cathode and, respectively, the FEA at ground potential. The latter mode of operation was used for in situ conditioning of the FEA. The DC gun is also equipped with a coaxial Faraday cup with $4 \mathrm{GHz}$ bandwidth and an emittance monitor. When $V_{\text {acc }}$ is larger than $-(10-20) \mathrm{kV}$, the FEA beam can go through the $1.5 \mathrm{~mm}$
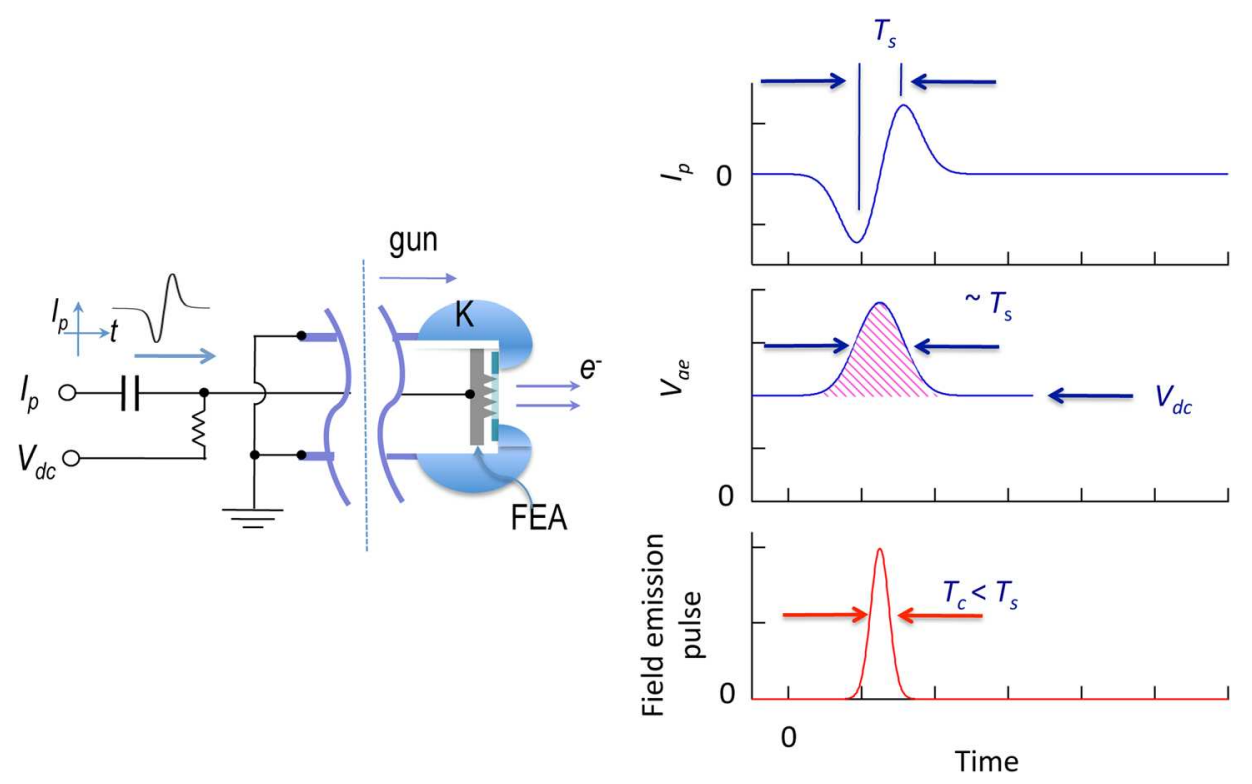

FIG. 2. (Color online) (Left) Schematic diagram of the picosecond electrical switching circuit of all metal FEAs by the bipolar current pulse method. FEA is mounted on a low-inductance FEA holder that is compatible with the acceleration field well above $30 \mathrm{MV} / \mathrm{m}$, cf. Figs. 3 and 4 . (Right) Concept of the bipolar current pulse switching method. The field emission current pulse (bottom) with the pulse duration of $T_{\mathrm{c}}$ is generated by modulating the gate-emitter potential with the duration of $T_{\mathrm{s}}$. $T_{\mathrm{s}}$ can be in picosecond range by applying bipolar current pulse. 


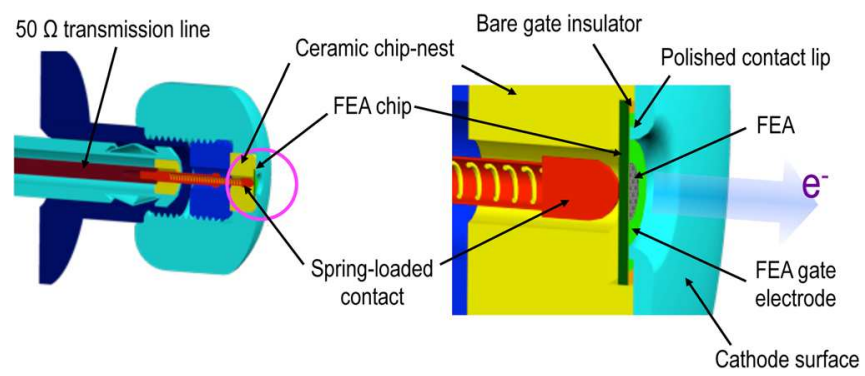

FIG. 3. (Color online) 3D CAD drawing of the low-inductance FEA holder. The right panel shows the enlarged view around FEA.

diameter anode iris. A solenoid integrated in the anode block is used to focus the electron beam in order to monitor it by a phosphor screen located at $\sim 500 \mathrm{~mm}$ downstream from the anode iris. A CCD camera captures the phosphor screen image of the emission pulse. Cathode flange is mechanically separated from the rest of the vacuum chamber by a flexible bellow and attached to a 3-axis motorized translation stage. This arrangement makes it possible to adjust remotely the relative position between the cathode and the anode. The distance between the FEA and the anode is variable between 5 and $25 \mathrm{~mm}$. In the experiment described below, the distance was set to $10 \mathrm{~mm}$. The field emission pulse waveform is detected by intercepting the beam with the Faraday cup and measured by a digital oscilloscope with $3 \mathrm{GHz}$ analogue bandwidth and $20 \mathrm{GS} / \mathrm{s}$ (Wavepro $7300 \mathrm{~A}$, LeCroy). We kept the repetition rate of the experiment at $100 \mathrm{~Hz}$ or below.

After loading the FEA into the DC gun, we conditioned it under high vacuum $\left(7 \times 10^{-9}\right.$ millibars $)$ and in neon gas environment with pressure of $1 \times 10^{-4}$ millibars. The conditioning was done by cycling the DC gate potential $V_{\text {ge }}$ between zero and a certain maximum value while monitoring the gate leak current and the field emission current $I_{\mathrm{a}}$ at the anode.
The gate leak current was less than $10 \%$ of $I_{\mathrm{a}}$, therefore, its effect on the $I_{\mathrm{a}}-V_{\text {ge }}$ relation was negligible. To condition the FEA at elevated emission current, the maximum $V_{\text {ge }}$ was gradually increased in time. Figure 6 shows two $I_{\mathrm{a}}-V_{\text {ge }}$ characteristics, one obtained after 4 days of the conditioning in high vacuum and another after 150 min of the neon gas conditioning. Both are represented well by the equation

$$
I_{\mathrm{a}}=A_{\mathrm{FN}}\left(V_{\mathrm{ge}} / B_{\mathrm{FN}}\right)^{2} \exp \left(-B_{\mathrm{FN}} / V_{\mathrm{ge}}\right)
$$

where the parameters $A_{\mathrm{FN}}$ and $B_{\mathrm{FN}}$ obtained from the fitting of measured $I_{\mathrm{a}}-V_{\mathrm{ge}}$ characteristics are summarized in Table I. The neon gas conditioning increased both $A_{\mathrm{FN}}$ and $B_{\mathrm{FN}}$. This fact is attributed to the increase of the number of active emitters together with the blunting of the sharpest emitters by bombardment of neon ions. ${ }^{15}$ This interpretation is also compatible with the improvement of the beam uniformity. The granularity of the beam image of Fig. 6(c), that was observed before the neon gas conditioning, diminished after the conditioning as shown in Fig. 6(d).

We also measured the $V_{\text {ge }}$ dependence of the field emission pulses. In this case, we applied bipolar current pulse and DC offset potential and varied the DC offset while having the amplitude of the bipolar pulses constant. To increase the pulsed emission current with a minimal DC emission background, we also applied a pulsed bias instead of the DC offset as shown in Fig. 8.

\section{EXPERIMENTAL RESULTS}

In Fig. 7(a), we show the field emission pulse waveform generated by exciting the FEA with bipolar current pulse on top of the DC gate potential offset $V_{\mathrm{ge}}{ }^{(\mathrm{dc})}$ equal to $32 \mathrm{~V}$ under $V_{\text {acc }}$ of $-40 \mathrm{kV}$. The waveform was measured by inserting the Faraday cup. The solenoid current $(\sim 1 \mathrm{~A})$ was
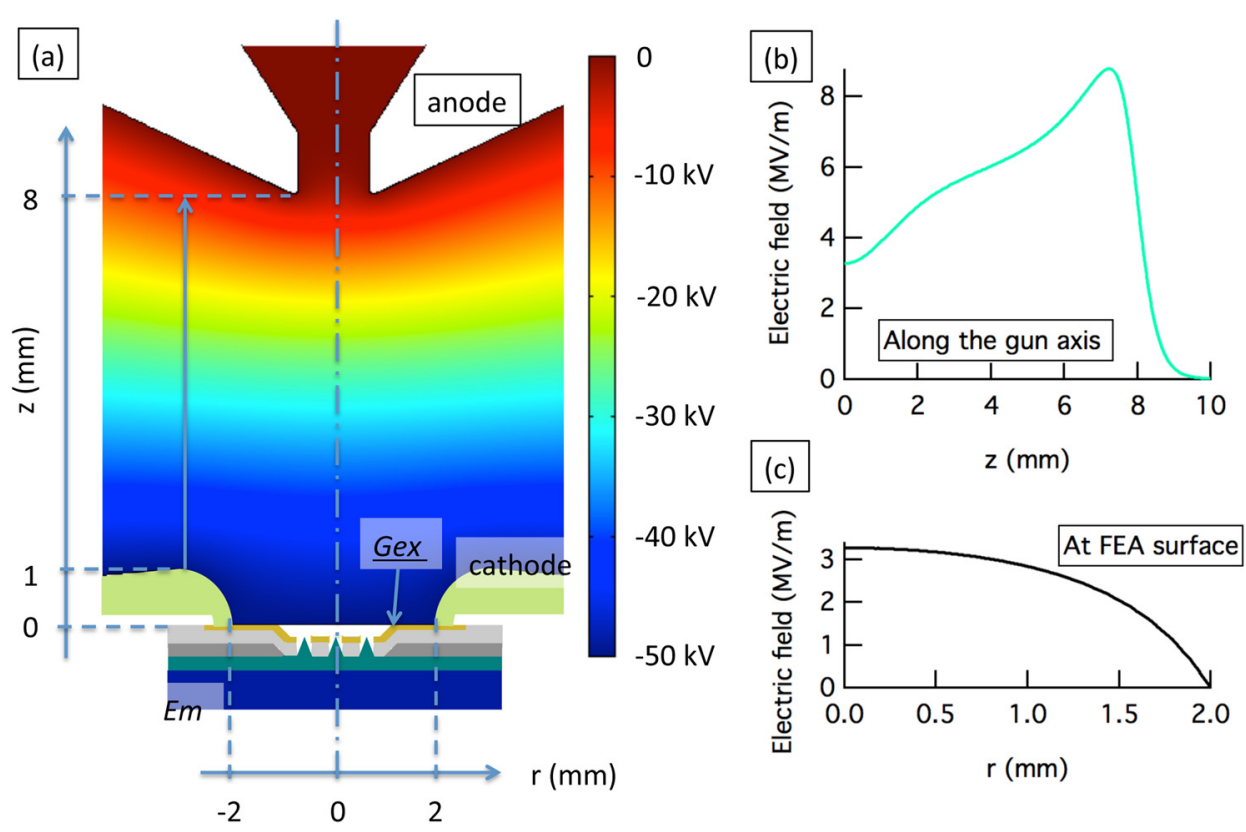

FIG. 4. (Color online) (a) Cross-section of the cathode-anode gap of the dc-gun teststand when the cathode is biased at - $50 \mathrm{kV}$ and the cathode-anode gap is $\sim 7 \mathrm{~mm}$. The color scale indicates the electrical potential distribution. (b) The electric field distribution along the axis of the gun. (c) The electric field distribution at the FEA surface. 


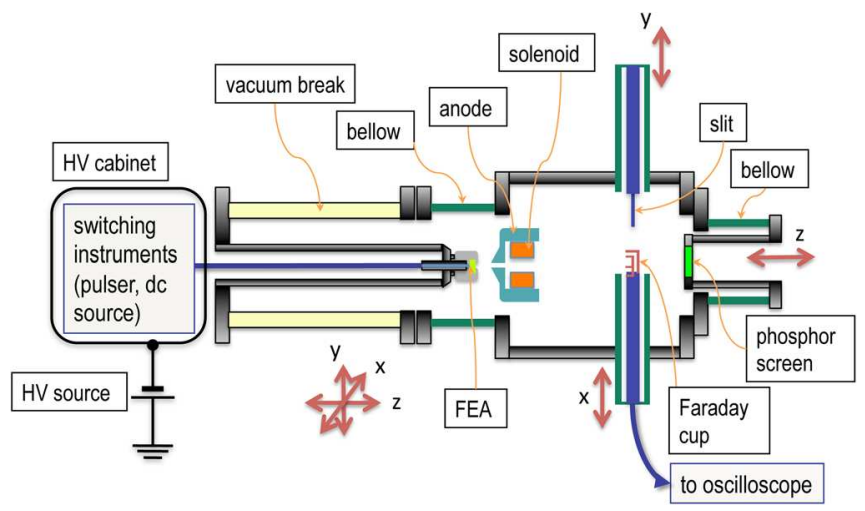

FIG. 5. (Color online) Schematic diagram of the DC gun teststand. The cathode is insulated from the gun chamber and biased by a DC voltage source together with the FEA switching instruments housed in the high voltage cabinet. The FEA beam that propagates through the anode can be focused by the solenoid, which is integrated in the anode block, on the phosphor screen, or on the Faraday cup when it is inserted. The slit assembly allows us to make the emittance measurement (cf. Ref. 6). The cathode-anode gap, the transverse displacement between FEA and the anode iris, as well as the position of the phosphor screen in the gun-axis direction, can be adjusted in situ. The anode is connected to either ground or a voltage supply via an electrical feedthrough.

adjusted to focus the beam on the Faraday cup. We observed field emission pulses with peak amplitude of $0.15 \mathrm{~mA}$ and pulse duration of $210 \mathrm{ps}$ FWHM. In Fig. 7(a), we also showed the simulated field emission pulse waveform. This was calculated by following steps: First, the gate potential pulse waveform $\delta V_{\text {ge }}(t)$ was calculated by integrating the bipolar current pulse applied to the gate capacitance of $0.25 \mathrm{nF}$. Then we calculated the field emission pulse $I_{\mathrm{a}}(t)$ by substituting the total gate potential $V_{\text {ge, }}$, which is given by the superposition of $V_{\mathrm{ge}}{ }^{(\mathrm{dc})}$ and $\delta V_{\mathrm{ge}}(t)$, into Eq. (1). The parameters $A_{\mathrm{FN}}$ and $B_{\mathrm{FN}}$ obtained from the DC measurements of the FEA after the end of the neon gas conditioning were used. The pulse parameters used in the calculation were the following: duration of the injection current pulses equal to 428 ps and the separation between the peaks of the pulses equal to $550 \mathrm{ps}$ [corresponding to $\Delta t$ equal to $(214 / \sqrt{2 \ln 2})$ ps and $T_{\mathrm{s}}$ equal to $550 \mathrm{ps}$, see Eq. (8) and related text below]. These values are in agreement with a separate measurement in a circuit that approximates the actual FEA experiment. The calculated waveform of the field emission pulse agreed well with experiment with the amplitude of the injection bipolar current pulses equal to $\sim 20 \mathrm{~A}$ (corresponding $\delta V_{\text {ge }}(t)$ amplitude of $\sim 32 \mathrm{~V}$ ). We also measured the relation between the field emission pulses and the gate potential by varying $V_{\mathrm{ge}}{ }^{(\mathrm{dc})}$ between -24 and $32 \mathrm{~V}$ under fixed pulsed gate potential amplitude. As shown in Fig. 6(b), this agreed well with the DC $I_{\mathrm{a}}-V_{\mathrm{ge}}$ characteristic.

As the DC $I_{\mathrm{a}}-V_{\text {ge }}$ characteristic in Fig. 7(b) shows, the maximum emission current after the conditioning was above $1 \mathrm{~mA}$ with a stable $I_{\mathrm{a}}-V_{\mathrm{ge}}$ relationship. To test the pulsed field emission for the peak current in the order of $1 \mathrm{~mA}$, the maximum gate potential has to be increased to $\sim 75 \mathrm{~V}$. We therefore replaced the DC offset voltage source with a slow pulsed voltage source with pulse duration of $\sim 3 \mu$ s, as shown
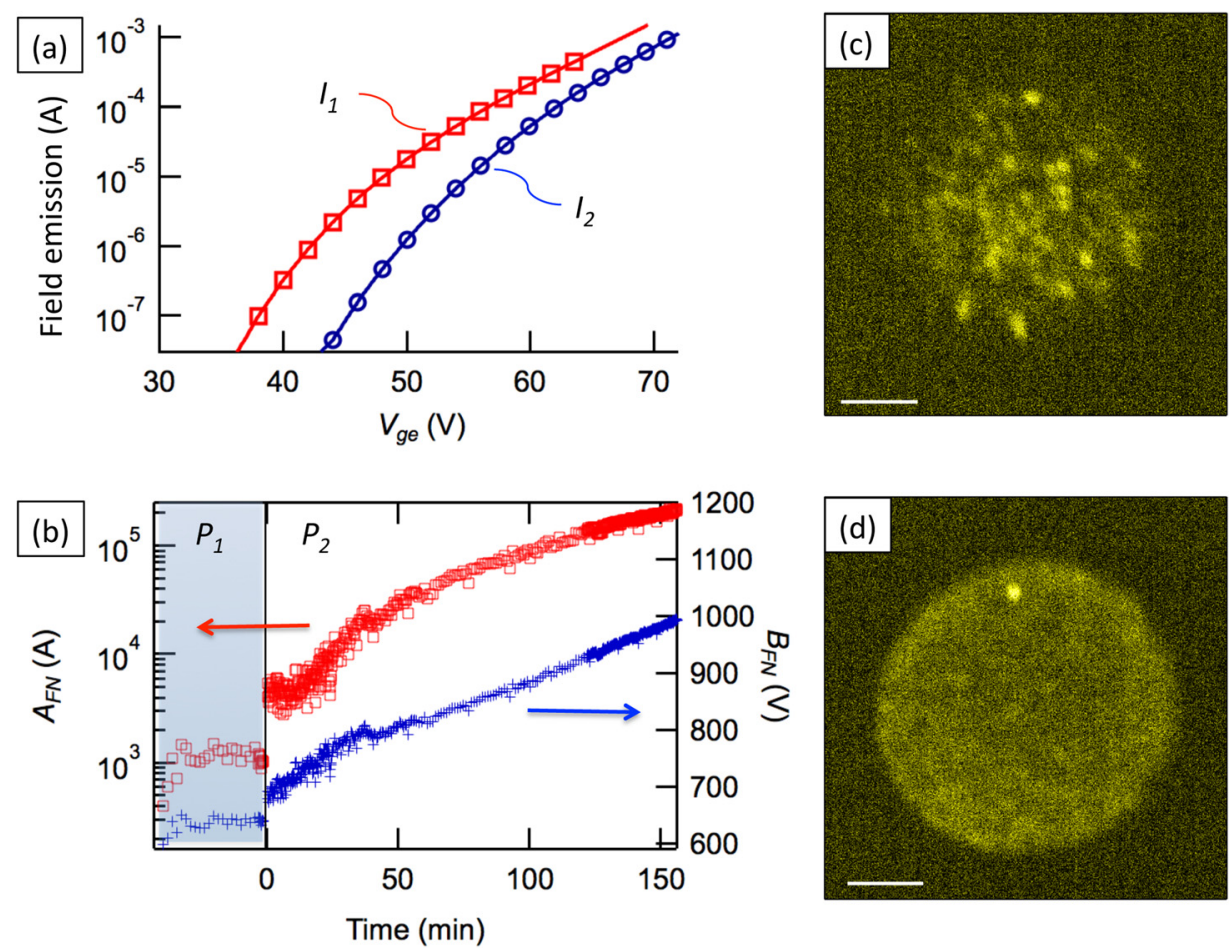

FIG. 6. (Color online) (a) Relation between the DC gate-emitter potential $V_{\text {ge }}$ and the emission current collected at anode, when the cathode was connected to ground and the anode potential was $1 \mathrm{kV} . I_{1}$ was obtained after 4 days of conditioning in UHV with the background pressure $P_{1}$ below $7 \times 10^{-9}$ millibars. $I_{2}$ was measured after 150 min conditioning in neon gas environment with the pressure of $10^{-4}$ millibars. (b) The evolution of the Fowler-Nordheim (FN) fitting parameter during the neon gas conditioning. (c) The beam image observed after the UHV conditioning. The FEA current pulse with $20 \mu \mathrm{A}$ amplitude and $200 \mathrm{~ns}$ duration was generated by applying $20 \mathrm{~V} \mathrm{DC}$ and $30 \mathrm{~V}$ pulse potential under $-20 \mathrm{kV}$ cathode potential. Solenoid current of $0.57 \mathrm{~A}$ was applied to image the beam distribution on the phosphor screen. (d) The beam image observed after the neon gas conditioning. The FEA current pulse was $20 \mu \mathrm{A}$ and $200 \mathrm{~ns}$ duration, generated by applying $20 \mathrm{DC}$ and $37 \mathrm{~V}$ pulse potential under the cathode potential of $-50 \mathrm{kV}$ (solenoid current was equal to $0.895 \mathrm{~A}$ ). 
TABLE I. Summary of the Fowler-Nordheim fitting of the current-voltage characteristics before and after the neon gas conditioning (Fig. 5).

\begin{tabular}{lcc}
\hline \hline Index & $A_{\mathrm{FN}}(\mathrm{A})$ & $B_{\mathrm{FN}}(\mathrm{V})$ \\
\hline$I_{1}$ & $1.5 \times 10^{3}$ & 650 \\
$I_{2}$ & $217 \times 10^{3}$ & 993 \\
\hline \hline
\end{tabular}

in Fig. 8(a). This way, we avoided the increased DC field emission background under high $V_{\text {acc }}$.

Using the slow pulse gate potential bias and the bipolar current pulsed excitation, the field emission pulse in Fig. 8(b) was observed. The field emission current amplitude of $1 \mathrm{~mA}$ matches well the expected value from the $I_{\mathrm{a}}-V_{\text {ge }}$ relationship. Observed pulse duration of $\sim 400$ ps FWHM is longer than the $0.15 \mathrm{~mA}$ pulse, due to the reduced electrical bandwidth of the switching system caused by the parasitic elements of the circuit that mixes the $\sim 3-\mu$ s-long offset pulse with the bipolar double pulse switching potential. Still, the fitting of the current pulse near the waveform peak showed the pulse duration of $260-300 \mathrm{ps}$ as in the case of $0.15 \mathrm{~mA}$ pulse.

\section{SIMULATION AT SHORT SWITCHING TIME SCALE}

The experiment showed that the bipolar current method can switch field emission of single-gate FEAs in subnanosecond time scale. Further reduction of the pulse duration down to tens of ps range might be possible, when a pulse generator with a shorter pulse duration and higher pulse amplitude is used. However, at shorter time scale, we need to take into account the finite propagation time that can cause the spatial distribution and dispersion of the switching potential and the field emission current across the FEA.

To have a quantitative estimate of the impact of the pulse propagation, we modeled the FEA as a distributed RLC circuit and analyzed the time dependence of the voltage $V(\vec{r}, t)$

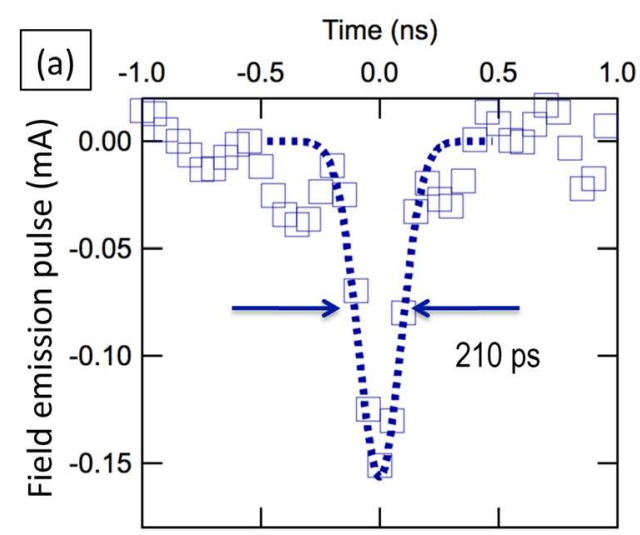

and the current density $\vec{J}(\vec{r}, t)$ by a numerical simulation. $V(\vec{r}, t)$ and $\vec{J}(\vec{r}, t)$ are the solutions of the following coupled partial differential equations:

$$
\begin{aligned}
& C \frac{\partial V(\vec{r}, t)}{\partial t}+\nabla \cdot \vec{J}(\vec{r}, t)=0, \\
& -\nabla V(\vec{r}, t)=R \vec{J}(\vec{r}, t)+L \frac{\partial \vec{J}(\vec{r}, t)}{\partial t},
\end{aligned}
$$

where $R, L$, and $C$ are, respectively, resistance, inductance, and capacitance per unit area given by the following [cf. Calame et al. ${ }^{16}$ ]:

$$
\begin{aligned}
& R=\left\{\begin{array}{l}
\frac{1}{\sigma}\left[\frac{1}{h_{\mathrm{g}}}\left(1-\frac{3 \pi r_{\mathrm{em}}^{2}}{p^{2}}\right)+\frac{1}{h_{\mathrm{s}}}\right]_{r<R_{\text {array }}} \\
\frac{1}{\sigma}\left[\frac{1}{h_{\mathrm{g}}}+\frac{1}{h_{\mathrm{s}}}\right]_{r<R_{\text {araay }}},
\end{array}\right. \\
& C=\left\{\begin{array}{c}
\frac{\varepsilon_{0} \varepsilon_{1}}{d}\left(1-\frac{3 \pi r_{\mathrm{em}}^{2}}{p^{2}}\right)+\left.\frac{3 C_{\text {tip }}}{p^{2}}\right|_{r<R_{\text {array }}} \\
\left.\frac{\varepsilon_{0}}{d}\left(\frac{\varepsilon_{1} \varepsilon_{2}}{\varepsilon_{1}+\varepsilon_{2}}\right)\right|_{r>R_{\text {array }}}, \\
f_{\mathrm{w}}=\left\{\left.\begin{array}{c}
\left.\frac{2 d}{1+f_{\mathrm{w}}}+\frac{h_{\mathrm{g}}}{2 f_{\mathrm{w}}}+\frac{h_{\mathrm{s}}}{2}\right), \\
\frac{1}{h_{\mathrm{g}}}+\frac{1}{h_{\mathrm{s}}}\left(1-\frac{3 \pi r_{\mathrm{em}}^{2}}{p^{2}}\right)^{-1}+\frac{1}{h_{\mathrm{s}}}
\end{array}\right|_{r<R_{\text {array }}}\right.
\end{array}\right.
\end{aligned}
$$

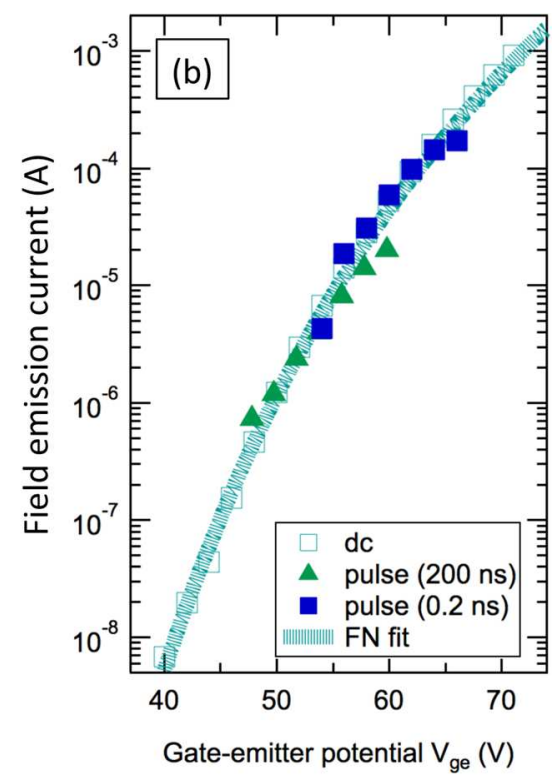

FIG. 7. (Color online) (a) Field emission pulse waveform generated by applying the bipolar current pulse on top of the DC bias ( $-32 \mathrm{~V}$ ) with cathode potential of $-40 \mathrm{kV}$. (b) Peak current amplitude of $0.2 \mathrm{~ns}$ pulses and $200 \mathrm{~ns}$ pulses, and DC emission in function of gate-emitter potential compared to the Fowler-Nordheim fitting (FN fit). 

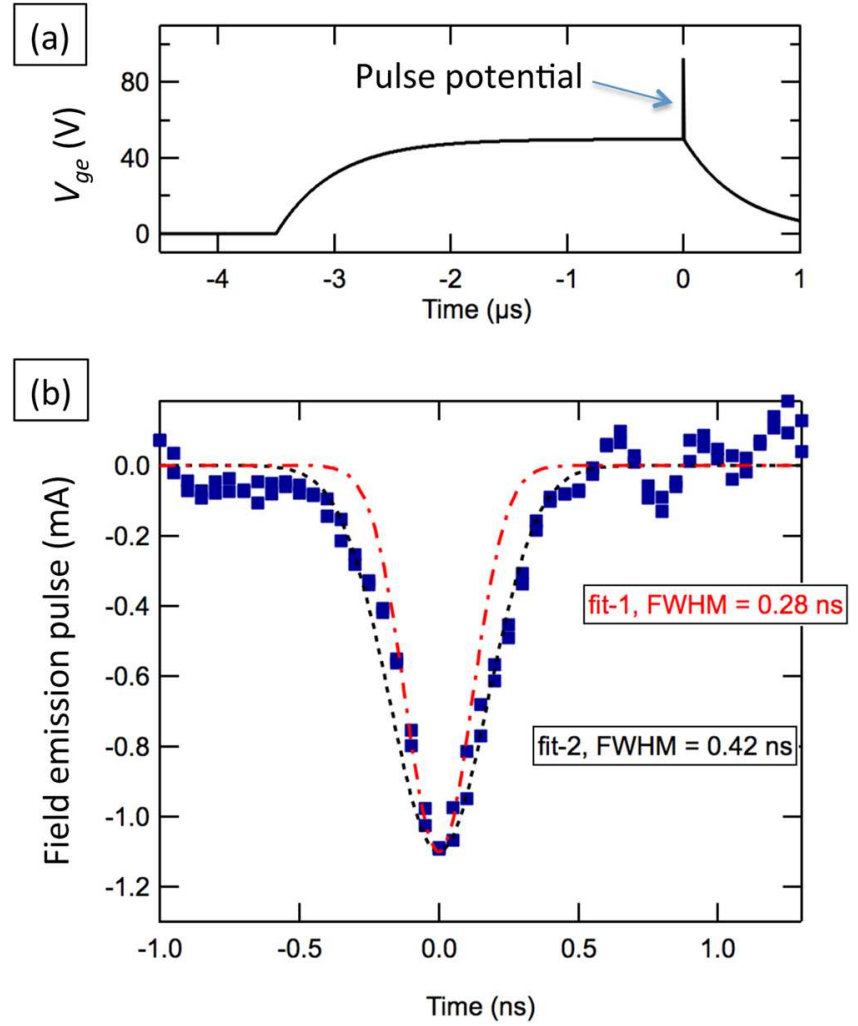

FIG. 8. (Color online) (a) Time evolution of the gate-emitter potential generated by super imposing a 3- $\mu$ s-long pulsed bias and picosecond switching pulse. (b) The current pulse $(1 \mathrm{~mA})$ generated by applying the gate-emitter potential depicted in (a). While Gaussian fitting of the entire current waveform (fit-2) gives pulse duration of 420 ps FWHM, the fitting around the pulse peak (fit-1) gives pulse duration of 280 ps FWHM.

In these equations, $h_{\mathrm{s}}$ is the effective substrate thickness, given by the skin depth at frequency $f$, equal to $\left(f \mu_{0} \sigma\right)^{-1 / 2}$, where $\mu_{0}$ is the vacuum permeability and $\sigma$ is the conductivity of the molybdenum film equal to $(20 \mu \Omega \mathrm{cm})^{-1}$ at room temperature. $\sigma$ was measured by van der Pau method for the sputtered molybdenum film we used as the emitter material ( $1 \mu \mathrm{m}$ thick) and the gate layer $(0.5 \mu \mathrm{m}$ thick). In the frequency range considered here (below $\sim 1 \mathrm{THz}$ ), $h_{\mathrm{g}}$ is given by the thickness of the gate layer $(0.5 \mu \mathrm{m})$ independent of the frequency, since it is thinner than the skin depth. In Eq. (5), $\varepsilon_{0}$ is the vacuum permittivity, $\varepsilon_{1}\left(\varepsilon_{2}\right)$ equal to 4.5 ( 6) is the dielectric constant of the $\mathrm{SiO}_{2}(\mathrm{SiON})$, and $d$ equal to $1.2 \mu \mathrm{m}$ is the insulator thickness within the array area $\left(r<R_{\text {array }}\right)$. Note that the total thickness of the insulator is two times thicker outside of the array area $\left(r>R_{\text {array }}\right) . r_{\mathrm{em}}$ equal to $1.45 \mu \mathrm{m}$ is the radius of the emitter cavity (where the $\mathrm{SiO}_{2}$ is removed), which is larger than the radius of the gate aperture equal to $\sim 1 \mu \mathrm{m}$. $C_{\text {tip }}$ is the tip capacitance equal to $0.11 \mathrm{fF}$, calculated by a finite element electrostatic solver (COMSOL Multiphysics ${ }^{17}$ ). $p$ is equal to $10 \mu \mathrm{m}$. The factor 3 takes into account the fact that every 4 th emitter aligned with the pitch of $5 \mu \mathrm{m}$ in the actual FEA was removed [see Fig. 1(b)] to secure the mechanical support of the gate layer by the insulator (in case of over etching). The expressions of $R$ and $L$ in Eqs. (5)-(7) take into account their correction by the emitter tip $\left(r<R_{\text {array }}\right)$.

To calculate the propagation of $V(\vec{r}, t)$ over the array, we numerically integrated Eqs. (2) and (3), by a finite element solver (COMSOL Multiphyiscs ${ }^{17}$ ). At the boundary, $r=r_{\text {edge }}$ equal to $2 \mathrm{~mm}$, we assumed the injection current density

$$
\begin{aligned}
\left.J(\vec{r}, t)\right|_{r=r_{\text {edge }}}= & \frac{I_{0}}{2 \pi r_{\text {edge }}}\left[\exp \left[-\left(\frac{t-t_{0}}{\sqrt{2} \Delta t}\right)^{2}\right]\right. \\
& \left.-\exp \left[-\left(\frac{t-t_{0}-T_{\mathrm{s}}}{\sqrt{2} \Delta t}\right)^{2}\right]\right],
\end{aligned}
$$

where $I_{0}$ is the amplitude, $T_{\mathrm{s}}$ is the separation between the two injection current pulses, $\Delta t$ is the rms pulse duration (FWHM duration equal to $2 \Delta t \sqrt{2 \ln 2}$ ), and $t_{0}$ is the offset in time.

Figure 9 shows thus calculated voltage propagation and the field emission current distribution induced by this voltage distribution. In this calculation, we assumed $t_{0}$ equal to $20 \mathrm{ps,}$
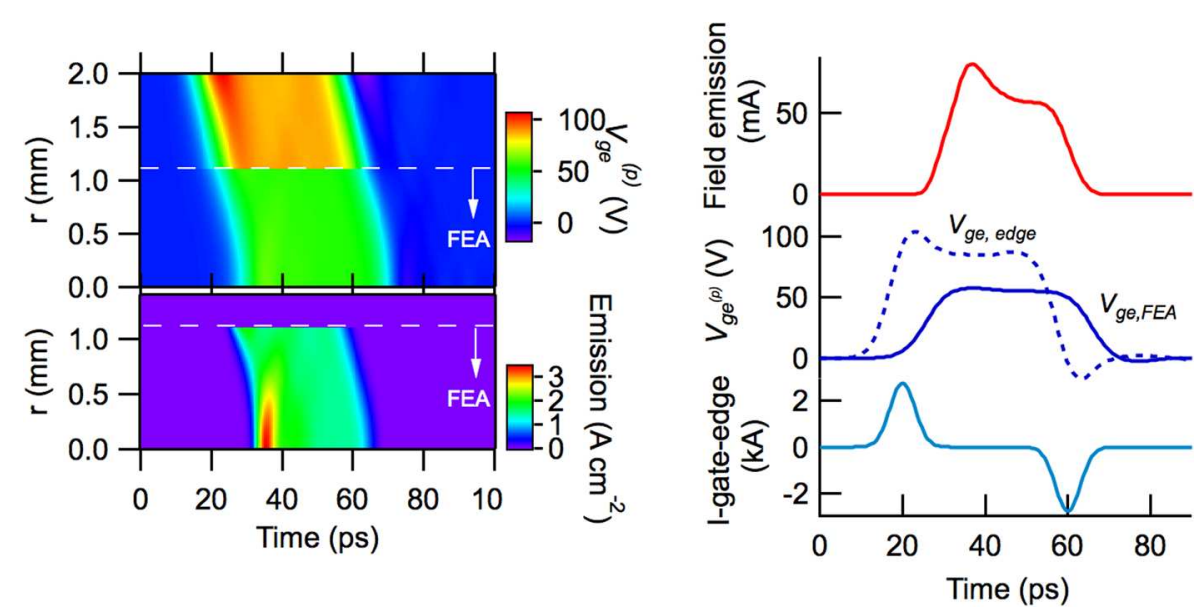

FIG. 9. (Color online) (a) Propagation of the gate-emitter potential pulse (top) and the induced field emission pulse (bottom) over the FEA excited by bipolar current pulse separated by $40 \mathrm{ps}$ at $r$ equal to $4 \mathrm{~mm}$. The array is below $r$ equal to $1.1 \mathrm{~mm}$. (b) Calculated total emission current (top), average pulse $V_{\text {ge }}$ (p) over the FEA $\left(V_{\mathrm{ge}, \mathrm{FEA}}\right.$, in the middle panel), and the pulse $V_{\mathrm{ge}}{ }^{(\mathrm{p})}$ at the edge of the gate $(r=2 \mathrm{~mm})$ excited by the bipolar current pulse injected at the edge of the gate $(r=2 \mathrm{~mm})$ shown in the bottom panel. DC offset of $40 \mathrm{~V}$ was assumed. 

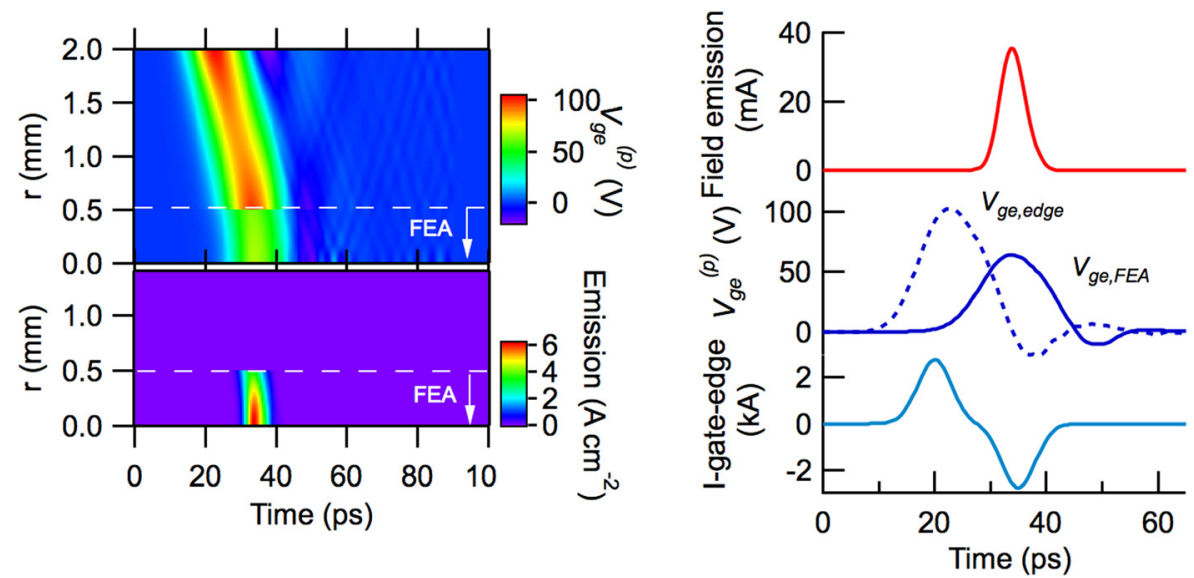

FIG. 10. (Color online) Same as Fig. 9 in the case of FEA with the diameter of $0.5 \mathrm{~mm}$ : (a) Propagation of the gate-emitter potential (top) and the induced field emission pulse (bottom) excited by bipolar current pulse separated by $15 \mathrm{ps}$. (b) Calculated total emission current (top), average pulse $V_{\mathrm{ge}}{ }^{(\mathrm{p})}$ over the FEA $\left(V_{\mathrm{ge}, \text { FEA }}\right.$, in the middle panel), and the pulse $V_{\mathrm{ge}}{ }^{(\mathrm{p})}$ at the edge of the gate $(r=2 \mathrm{~mm})$ excited by the bipolar current pulse injected at the edge of the gate $(r=2 \mathrm{~mm})$ shown in the bottom panel.

FWHM current pulse duration of $10 \mathrm{ps}(\Delta t$ equal to $5 / \sqrt{2 \ln 2}$ ps), $T_{\mathrm{s}}$ equal to $40 \mathrm{ps}$, and $I_{0}$ equal to $2.7 \mathrm{kA}$. We assumed $f$ equal to $100 \mathrm{GHz}$ to calculate $h_{\mathrm{s}}$. We assumed a DC offset of $40 \mathrm{~V}$ and the potential pulse amplitude of $55 \mathrm{~V}$ [see Fig. 9(c)] within the FEA. These values and the predicted field emission pulse amplitude (with the average tip current of $\sim 1 \mu \mathrm{A}$ ) are within the reported parameter range of the all metal single-gate FEAs considered here (after the neon gas conditioning) as shown in previous experiments. ${ }^{15}$ The potential $V_{\text {ge,edge }}$ at $r_{\text {edge }}(=2 \mathrm{~mm})$ with duration of $\sim 40$ ps propagates to the center of the FEA in $\sim 20 \mathrm{ps}$ as shown in the upper panel of Fig. 9(a). Combining the calculated gate potential distribution $V_{\text {ge }}(\vec{r}, t)$ with the empirical emission characteristic Eq. (1) with the coefficients obtained after the neon gas conditioning, the field emission distribution shown in the bottom panel of Fig. 9(a) was calculated. Except an overshoot in time near the center of the FEA, a spatially uniform current distribution is obtained. The current pulse waveform of the total field emission pulse is obtained by integrating the field emission distribution over the FEA area. For this 2-mm-diameter FEA at the assumed switching condition, the amplitude of the current pulse is equal to $\sim 80 \mathrm{~mA}$ and the pulse duration of the total field emission pulse is equal to $\sim 30 \mathrm{ps}$, see Fig. 9(b).

By reducing the FEA diameter to $1 \mathrm{~mm}$ and $T_{\mathrm{s}}$ to $15 \mathrm{ps}$, the potential and the field emission distributions shown in Fig. 10(a) were calculated. Near the center of the FEA, a slight concentration of the potential due to the circular geometry enhanced the current density by $20 \%$. As shown in Fig. 10(b), the amplitude of the total field emission pulse is $\sim 35 \mathrm{~mA}$, which is smaller than the previous case because of the smaller FEA area. The pulse duration is now reduced to 5 ps.

We note that the here demonstrated short switching time of 5-30 ps by the bipolar current method is not apparent. This is because the method implicitly assumed that the gate electrode is represented as a lumped capacitor; therefore, the case when the switching voltage propagation time over the FEA is comparable to the duration of the excitation pulses was not assumed. We attribute the fact that the method works down to $\sim 5$ ps as shown in Fig. 10 to the sufficient dumping of the oscillation of the potential wave by the electrical loss of the gate and the emitter layers. As such, it appears to be difficult to extend the method at shorter time scale because of the insufficient dumping. In fact, calculation at shorter $\Delta t$ and $T_{\mathrm{s}}$ (not shown) resulted in degradation of the spatial uniformity and oscillating temporal field emission distribution. Further reduction of $T_{\mathrm{s}}$ while keeping the same $\Delta t$ decreases the pulse duration but with the decrease of the pulse amplitude at the same time; therefore, it is not advantageous.

\section{SUMMARY AND CONCLUSION}

To generate fast electron pulses using single-gate FEAs, we proposed the bipolar current method. By experimentally testing the method with our all metal FEAs in the DC gun teststand, we showed that it is possible to switch FEAs in high acceleration field within $\sim 210 \mathrm{ps}$. This is comparable to the pulse duration of 130 ps observed for the laser-excited field emission pulses from semiconductor FEAs. ${ }^{18}$ The emission current amplitude and the pulse duration in the present experiment were limited, on one hand by the pulse parameters of the high voltage pulse generator used to drive the FEA and on the other by the finite propagation time of the gate potential pulse over the FEA. By a numerical modeling that takes into account the latter effect, we found that it is theoretically possible to generate electron pulses with $\sim 5$ ps duration from $\sim 1$-mm-diameter FEAs. When such short electron pulses ( $\sim 5$ ps FWHM) are practically realized, it is feasible to use these FEAs as electron sources for an RF electron gun operating at $1.5 \mathrm{GHz}$ since the relative longitudinal energy spread due to the finite electron bunch length will be limited to $3 \times 10^{-4}$.

The required high level of the gate injection current, especially at short time scale, can be a cause of the device failure for long term operation due to joule heating and electromigration. ${ }^{19}$ Owing to the metal substrate and the low substrate resistance of the FEA, the estimated peak dissipation power 
upon switching at $\sim 200$ ps is below $\sim 20 \mathrm{~W}$ despite the high level of the estimated injection current density at $r_{\text {edge }}$ equal to $\sim 10^{5} \mathrm{~A} \mathrm{~cm}^{-2}$. Therefore, for the low duty cycle operation $\left(<10^{-7}\right.$, with the repetition rate below $\left.100 \mathrm{~Hz}\right)$ as used in the experiment, the power dissipation and the electromigration may not be limiting factors for the $\sim 5$ ps switching operation of FEAs. However, reduction of the total gate capacitance by an order of magnitude by modifying the FEA structure, e.g., as the one similar to the low-capacitance Spindt FEA ${ }^{1}$ will be advantageous since the required excitation injection current will decrease proportionally to the total gate capacitance.

Finally, we note that the switching method considered here is also applicable to all metal double-gate FEAs. ${ }^{8}$ Since the switching of the FEA current requires only the modulation of the electron extraction potential between the first gate electrode and the emitter substrate and the potential between the electron collimation gate and the electron extraction gate electrode stays constant, the second gate electrode for the beam collimation does not increase the load capacitance to switch the field emission pulse. However, the necessity of an additional contact constitutes a nontrivial technical challenge, in order to minimize the additional parasitic inductance and capacitance in the system. This will require a careful design study in order to realize such 5 ps electrical gating.

\section{ACKNOWLEDGMENTS}

The authors acknowledge B. Haas, J. Leemann, A. Luecke, E. Kirk, and T. Vogel for their help for FEA fabrication, C. Zumbach, S. Vosar, and B. Scheidegger for their technical support for upgrading the mechanics of the teststand (translation stage, laser port, cathode flange rail, and beam monitors), S. C. Leemann, M. Dach, C. Higgs, and T. Vogel for their support to reestablish and upgrade the operation of the DC gun teststand, and H.-H. Braun, J. Gobrecht, A. Wrulich, and C. Gough for their support and helpful discussions. This work was conducted within the SwissFEL X-ray free-electron laser project at the Paul Scherrer Institute. This work was partially supported by Swiss National Science Foundation, Nos. 200020_143428 and 200021_147101.

${ }^{1}$ D. R. Whaley, B. M. Gannon, V. O. Heinen, K. E. Kreischer, C. E. Holland, and C. A. Spindt, IEEE Trans. Plasma Sci. 30, 998 (2002).

${ }^{2}$ S. Tsujino, M. Paraliev, E. Kirk, C. Gough, S. Ivkovic, and H.-H. Braun, Phys. Plasmas 18, 064502 (2011).

${ }^{3}$ S. Tsujino, M. Paraliev, E. Kirk, T. Vogel, F. Le Pimpec, C. Gough, S. Ivkovic, and H.-H. Braun, J. Vac. Sci. Technol. B 29, $02 B 117$ (2011).

${ }^{4}$ S. Tsujino, M. Paraliev, P. Helfenstein, and H. H. Braun, Paper Presented at 25th International Vacuum Nanoelectronics Conference (IVNC), Jeju, Korea, 2012 (unpublished).

${ }^{5}$ S. Tsujino, M. Paraliev, E. Kirk, and H. H. Braun, Paper Presented at International Vacuum Electronics and Vacuum Electron Sources Conference (IVEC-IVESC 2012), Monterey, CA, 2012 (unpublished).

${ }^{6}$ S. C. Leemann, A. Streun, and A. F. Wrulich, Phys. Rev. ST Accel. Beams 10, 071302 (2007).

${ }^{7}$ M. Dehler, A. Candel, and E. Gjonaj, J. Vac. Sci. Technol. B 24, 892 (2006).

${ }^{8}$ P. Helfenstein, V. A. Guzenko, H. W. Fink, and S. Tsujino, J. Appl. Phys. 113, 043306 (2013).

${ }^{9}$ M. Reiser, Theory, Design of Charged Particle Beams (WILEY-VCH Verlag GmbH \& Co., Weinheim, 2008).

${ }^{10}$ J. Teichert, German-Turkish Workshop on Superconducting Accelerators for FEL- and Bremsstrahlung Applications, Antalya, Turkey, 2011.

${ }^{11}$ E. Kirk, S. Tsujino, T. Vogel, K. Jefimovs, J. Gobrecht, and A. F. Wrulich, J. Vac. Sci. Technol. B 27, 1813 (2009).

${ }^{12}$ M. Paraliev, C. Gough, S. Ivkovic, and F. Le Pimpec, Paper Presented at the IEEE International Power Modulator and High Voltage Conference 2010 (IPMHVC 2010), Atlanta, GA, USA, 2010 (unpublished).

${ }^{13}$ M. Paraliev, S. Tsujino, C. Gough, E. Kirk, and S. Ivkovic, Paper Presented at IEEE Pulsed Power Conference (PPC), Chicago, IL, 2011 (unpublished).

${ }^{14}$ See http://www.fidtechnology.com/products/fpg-picosecond.html for FID $\mathrm{GmbH}$.

${ }^{15}$ S. Tsujino, M. Paraliev, E. Kirk, and H.-H. Braun, Appl. Phys. Lett. 99, 073101 (2011).

${ }^{16}$ J. P. Calame, H. F. Gray, and J. L. Shaw, J. Appl. Phys. 73, 1485 (1993).

${ }^{17}$ See http://www.comsol.com for COMSOL Multiphysics.

${ }^{18}$ C.-J. Chiang, K. X. Liu, and J. P. Heritage, Appl. Phys. Lett. 90, 083506 (2007).

${ }^{19}$ Arijit Roy and Cher Ming Tan, J. Appl. Phys. 103, 093707 (2008). 